\title{
Virtual affective consciousness and raw social AI
}

\author{
W. Pan \& Y. Yang \\ Sichuan University, Chengdu, Sichuan Province, China
}

\begin{abstract}
In this paper, we attempt to philosophically eliminate the conceptual problems in developing affective artificial agents, and then put forward an industry and research direction called Raw Social AI. We first provide a set of laws for affective concepts understanding, and then suggest that we should build unconscious concepts with uncertainty. We address the problem of qualia supply, and discuss how to implement the initiative of affective agents. We suggest that initiative be the cue of utility of affective agents. We advocate that the initiative of affective agents must be realized as social services. Raw social AI is about using AI to record, model and predict people's emotional reactions in the real or virtual world. It is necessary for us to establish the infrastructure of raw social AI.
\end{abstract}

\section{GENERAL INSTRUCTIONS}

As a cognitive AI direction, affective computing is different from neuropsychology (Berlucchi 2017) and neurosociology (Franks 2019). The latter two have to link people's mental performance and behaviors to brain organizations, and this is often related to understanding the symptoms of people with brain damage and the medical needs of curing the patients. The AI direction does not necessarily have to simulate the human brain and neural mechanisms, but to realize and surpass human abilities, mainly intelligence. Recognizing emotions, as a kind of human cognitive ability, is also a kind of ability that AI needs to realize and surpass through computing. As discussed in the book (Franks 2019), emotional performance belongs to the "unconscious" process of people, and it is very difficult to track and discover by exploring brain nerve activities (such as brain waves and fMRI scans). But human emotions can always be correctly recognized by themselves and others to a considerable extent. For example, one can recognize other people's emotions by observing their facial expressions. This ability can also be imitated and surpassed by AI. Training artificial intelligence and algorithms to recognize facial emotions has been quite successful (Sariyanidi et al. 2015). Microsoft has provided emotional computing services through the Azure cloud platform, that is, to recognize human emotions through the facial expressions of people in pictures and videos (Microsoft 2021). Exciting progress has also been made in the recognition of emotions from language text (Calvo \& Kim 2013), body gestures (Castellano et al. 2007) and paralinguistics features (such as pitch and rhythm) (Schuller \& Batliner 2013).

Emotional computing has important applications in human-computer interaction and virtual world actions. As reflected in papers published in the new book about affective factors in humancomputer interaction (Jeon 2017a) and IEEE Journal of Affective Computing (IEEE 2021), people are becoming more and more interested in understanding, designing, and evaluating the use of emotions and affects in socio-technical systems. There are many uses for developing affective artificial agents (Scheutz 2012). Emotions and affects recognition through facial expressions, gestures, speech, and cognitive processes make it possible to construct emotions as important user inputs of the system (Hudlicka 2017). Emotional elements also allow a more comprehensive understanding of human-machine systems (Jeon 2017b). The goal of affective computing is to 
design a computer system that can recognize and express affects, have emotions and use them in decision-making in the real or virtual world (Picard 2010).

However, there are several obvious obstacles to the development of affective computing. The article (Scheutz 2012) summarizes them as conceptual challenges, architectural and implementation challenges, and evaluation challenges. This judgment can still apply to the current situation. Although there have been several theories of computational emotion modeling (Reisenzein et al. 2013), such as discrete or categorical theory, dimensionality theory, and component theory, people cannot fully agree with the cognitive concept of influence there. For example, people cannot clearly distinguish the concepts of emotion, affect, mood and feeling, so it is more difficult to design affective agents to express them correctly. Although the agent's affective control mechanism can be beneficial to the agent's performance, there is a lack of research that connects the emotional control mechanism with the agent's work and realizes its utility. Sometimes, these problems lead to an embodied view of emotion and affect, together with other cognition problem (Shapiro 2014, Borghi \& Binkofski 2014). More complex analysis methods have also emerged: one is multimodal affect analysis, such as multimodal affect analysis using audio, visual and text information (Poria et al. 2017), and the other is sentiment time series analysis. In order to build artificial intelligence that understands human affects, modeling affective dynamics is an important direction. But there are still great difficulties. The latest paper (Ong et al. 2021) believes that the biggest obstacle to be overcome in this regard is the inherent difficulty of constructing computational time series models, as well as the difficulty and high cost of collecting high-quality data sets. There are several existing time series emotional data sets, such as the RECOLA data set (Ringeval et al. 2013) and the AM-FED data set (McDuff et al. 2013). The former is an original collaboration and affective interaction database; the latter is a video containing 242 people watching advertisements at home. The Stanford Emotional Narrative Data Set (SEND) (Ong et al. 2021) is a new data set containing annotated videos of unscripted autobiographical emotional narratives, and includes RNN-LSTM, HMM and VRNN modeling of emotional time series.

Augmented reality (AR) and mixed reality (XR) are the next generation computing platforms. We will rely heavily on the virtual world connected to the real world. Emotional preference is the key to actual decision-making (Cozolino 2006). Using AI to record, modelling and prediction people's emotional reactions in the real and virtual world is raw social AI.

In this paper, we attempt to philosophically eliminate the confusion in developing affective artificial agents. We suggest that consensus on intrapsychic cognitive concepts about emotion and affect is impossible, and there is no necessary for us to develop affective agents on that consensus. We first provide a set of laws for unconscious concepts understanding, which are the laws of selfillustration, initiative, deferent names, and secondhand. Then, we address the problem of affective qualia supply, then we discuss how to implement the initiative of affective agents and suggests that initiative be the cue of utility of affective agents. Finally, we point out that the affective agents cannot have initiative unless the initiative is provided as services.

\section{THE CODE FOR AFFECTIVE UNCONSCIOUS}

There are still some philosophical issues in AI, e.g., consciousness, unconscious and meaning, but AI researchers do not have to leave these philosophical questions to philosophers. As far as philosophical issues are concerned in AI, past thinkers are very profound. We think there are four laws for affective unconscious understanding.

\subsection{The law of self-illustration}

Some philosophers think that mind has qualitative conscious states, which is called qualia. Following Searle (Searle, 2004, p84-85), qualia are the inner experiences which cannot have a functionalism account, as argued in the old thought experiment spectrum inversion. We use the law of self-illustration to name the hypothesis that the inner states and experiences in one's mind cannot be directly detected. Note the neural mechanisms research (Aday et al. 2017) cannot falsify this law. We cannot detect the qualia of color by examining the neural system in the brain, as argued in spectrum inversion. 
Hence the observations about mind of any great ancient thinkers are as valuable as the descriptions given by modern philosophers, psychologists and scientists. I will then refer some Chinese ancient thinkers' discourses in this paper.

\subsection{The law of initiative}

Some people think that without determinism, the exploration of decision-making models will be meaningless (Franks 2019). Though one can receive requirements and commands from others, the mind initiate thinking and body behavior by itself. Sometimes, one's mind can be significantly affected by other peoples or things, but we should think the minds hold its initiative except suffering illness. Chinese ancient thinker Xun Zi (荀子, 313 BC - 238 BC) wrote:

“ (心者) 出令而无所受令, 自禁也, 自使也, 自夺也, 自取也, 自行也, 自止也。” These sentences are copied from the book (Jue Zhang 2012). These sentences can be translated as:

"The mind issued orders but did not accept orders, and it prohibits and drives to do something on its own, discards and accepts things on its own, acts and stops on its own."

In dictionary, initiative is "the ability to decide and act on your own without waiting for somebody to tell you what to do." Follow the great thinker Xun Zi, we must acknowledge that initiative is the most important trait of mind and virtual mind.

\subsection{The law of different names}

By the law of undetectability, any object in mind can-not be detected by others. Therefore, if two objects with different names have the very same relation, at the very least through the language description, with other objects concerned, the two objects will be considered having same concept position in conceptual system but have different names. Chinese ancient philosopher Lao Zi (老 子, about $571 \mathrm{BC}-471 \mathrm{BC}$ ) wrote:

“无, 名万物之始, 有, 名万物之母……此两者, 同出而异名, 同谓之玄。”

These sentences are copied from the currently edited and annotated book (Shangkuan Rao 2016).

These sentences can be translated as:

"Wu (nothing) is used to name the beginning of all things, You (being) is used to the name of the mother of all things... Both of Wu and You are originated identically but they are different names, they together are called as Xuan.”

Lao $\mathrm{Zi}$ described that $\mathrm{Wu}$ and You has same position in the conceptual system, i.e., they both connected with all things, but they have different name.

We use the law of different names to address that the difference of some concepts cannot clearly specified in a conceptual system, especially when we want to build the cognitive model of mind. The inner of a mind is undetectable; thus, we only can relate them with outer objects and processes.

\subsection{The law of secondhand}

Many mental states have intentionality, i.e., "they are directed at or about or of objects and affairs in the world", and "meaning is one kind of intentionality" (Searle 1983). The world for a specified mind really contains the inner world of the mind. Once one is thinking meaning intentionality itself, the intension then is direct at the meaning itself but not what the meaning intends. We never can talk the real objects, we just talk the meaning and intent of the objects, even when we have not perceived the objects. The intent is the fakeness when it is intent to an intent, for the latter is undetectable, thus it can never be really understood or known. Which we call as the law of fakeness. Chinese ancient thinker Gongsunlong Zi (公孙龙子, 320 BC - 250 BC) wrote:

“物莫非指, 而指非指。”

This sentence is copied from the currently edited and annotated book (Kejian Huang 2012). This sentence can be translated as:

"Things are all intended or pointed (in mind), but the intending or pointing are not intended or pointed (in mind)."

And in the Chinese Tang Dynasty, Zen Master Xuanjue (玄觉, AD $665 \sim$ AD 713) wrote:

“夫以知知物, 物在知亦在; 若以知知知, 知知则离物。” 
These sentences can be found in the 38th volume of the currently edited book (Jifu Lan 2004 p65). I just only modified the punctuations. These sentences can be translated as:

"When one exploits his/her knowing ability to know some object, the knowing exists when the object exist; when one exploits his/her knowing ability to know the knowing, the knowing of knowing object have detached from the object."

Some other thinkers in ancient China also expressed similar perspectives and experiences. The knowing of knowing is just the consciousness. Now we know that unconscious processes take place hundreds of milliseconds before consciousness (Franks 2019).

\section{IS EVERYTHING MEANINGLESS?}

Does these laws mean that everything is meaningless? This simply means that the ultimate meaning we mean in language and consciousness is the qualia.

Meaning is not knowledge; the semantics of words ultimately point to meaning. There is no definition of purely cognitively abstract natural language words. These terms, such as "meaning", "intelligence", "consciousness", "value", "beauty" and "time", cannot be defined as ontologies, objects, processes, indexes, etc. Completely defined These words must use the meaning of the words themselves, and therefore can only be defined by cycles, and there are no static definitions of them. Meaning is the reflection of self-consciousness. When we talk about affect, we really talk about the meaning of affect. The meaning of affect is mirrored in mind.

The ontologies, which are developed in AI, which are used to defined what things in world are, cannot be directly related to the qualia. The real consolidation of the natural language ontologies is impossible. The Ontologies are really related to meaning, they capture meaning with the ontological connections of words.

We can simply think that through ontologies the qualia are combined into meaning. When we are talking or reading, meaning does not directly convey to each other. We deliver the connections in ontologies to each other, and then one's inner qualia are combined again. We don't know whether there are the same qualia or the same number of qualia in different people's consciousness.

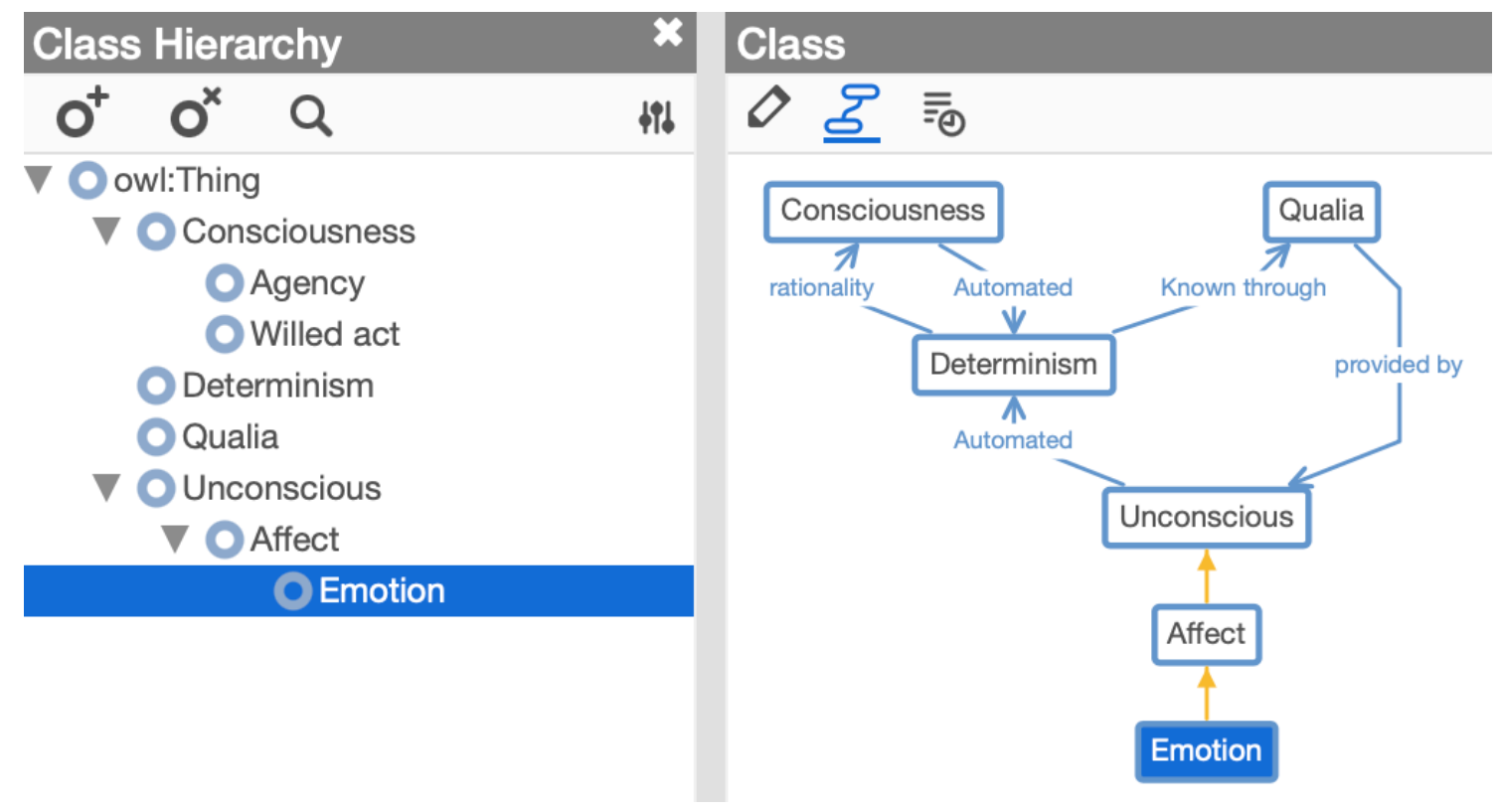

Figure 1. A simple knowledge graph of affect without consensus

\section{UNCERTAIN KNOWLEDGE GRAPH OF AFFECT}

Any description of mind is intentionality intrapsychic, the intentioned is not directly detectable now. Even though, we can express some temporarily conceived theorems, proofs and definitions. We call these kind of theorems, proofs and definitions are fake. For example: 
Fake Theorem: intentionality has qualia.

Fake Proof: The qualia is the inner experiences, and the intentionality can be experienced, hence the intentionality has qualia.

Fake Definition: The quale of intentionality is called qualintent.

Although I can edit a knowledge graph about affect as shown in Figure 1, no one will completely agree with the conceptual relationship, and it is difficult for anyone to completely refute it. The conceptual relationship between consciousness and affect is always changing. For example, when the unconscious is not considered, the concept of consciousness actually includes the unconscious. When agency and reason are connected, they are actually opposed to willed act. It is impossible for us to get the unchanging relationship of these concepts.

\section{AFFECTIVE QUALIA SUPPLY}

With the advancement of AI and computing technology as augmented reality, people will increasingly live in a virtual world, as in the movie "Ready Player One." This brings back two problems. One is the lack of real-world experience, and the other is that the virtual world may be different. These problems may be called qualia lack and qualia disorder. There are three techniques that are useful for qualia consistence and qualia supply:

1 Knowledge graph and ontologies. They can be used to supply connection of qualia.

2 Virtual reality, AR and mixed reality. They can be used to build qualia.

3 Initiative Service. This is the key ability of human-like agents.

\section{THE RAW SOCIAL AI: INITIATIVE AS SOCIAL SERVICES}

Any AI agent is simply a computational machine that makes calculations and controls actions based on inputs and perceived information. The computational mechanism of an AI agent is predesigned and evolutionary rules are predetermined even if it evolves. So how can the machine have the human like initiative?

However, a person does not live alone, she or he lives in society, i.e., the whole of countless people. And the society does not exist temporarily or appear recently, it exists historically. The societies have their civilizations which are merely the result of the overall human history. That will be a very difficult endeavor to let the AI agents understand the whole society, history, and civilizations. We cannot pin our hopes on possible success of that.

Therefore, an AI agent cannot be initiative on its own. And if an AI agent generates certainty from certain user inputs, it cannot be perceived as initiative. If an AI agent is initiative, its behavior is not defined for its human users, owners, or friends, but it is what they need.

Psychologists think that human actions are all related to their emotion system. Emotion and affect of people are concerned with all aspects of situation assessment and belief information, including decision making. However, in my opinion, in the most depth of utility, emotion and affect is used to feedback social value. Emotions and affects are interactive at the level of society, just as fairness and efficiency can be "calculated" at the level of society.

The artificial affective agents cannot have initiative unless the initiative is provided as services. Fortunately, there is now a choice for initiative: use a distributed consensus mechanism such as the PoS consensus mechanism (Fairley 2018) to decide whether to act.

\section{CONCLUSION}

For us to successively build the connection between real world and virtual environment, it is necessary to establish the infrastructure of raw social AI, conceptualized uncertainly and implemented technically.

Corresponding author: Wuming Pan (panwuming@scu.edu.cn)

\section{REFERENCES}

Aday, J., Rizer, W. \& Carlson, J. M. 2017. Neural Mechanisms of Emotions and Affect. In Emotions and Affect in Human Factors and Human-Computer Interaction, Pages 27-88, Mounghoon Jeon, eds., 
Academic Press, London, San Diego, Cam-bridge, Oxford.

Berlucchi, G. 2017. Neuropsychology, Reference Module in Neuroscience and Biobehavioral Psychology, Elsevier.

Borghi A. M. \& Binkofski, F. 2014. Words as Social Tools: An Embodied View on Abstract Concepts. Springer.

Calvo, R. A. \& Kim, S. M. 2013. Emotions in text: Dimensional and categorical models, Comput. Intell., vol. 29 , no. 3 , pp. 527-543.

Castellano, G., Villalba, S. D. \& Camurri, A. 2007. Recognising human emotions from body movement and gesture dynamics, Proc. Int. Conf. Affect. Comput. Intell. Interaction, pp. 71-82.

Cozolino, L. 2006. The neuroscience of human relationships: Attachment and the developing social brain. London: Norton Press.

Fairley, P. 2018. Ethereum will cut back its absurd energy use. IEEE spectrum 56.1, 29-32.

Franks, D. 2019. Neurosociology: Fundamentals and Current Findings. Springer.

Hudlicka, E. 2017. The Computational Modeling of Cognition-Emotion Interactions: Theoretical and Practical Relevance for Behavioral Healthcare. In Emotions and Affect in Human Factors and Human-Computer Interaction, Pages 383-436, Jeon, M. eds., Academic Press, London, San Diego, Cambridge, Oxford.

IEEE. 2021. IEEE Transactions on Affective Computing. https://www.computer.org/csdl/journal/ta.

Jeon, M. 2017a. (eds) Emotions and Affect in Human Factors and Human-Computer Interaction. Academic Press, London, San Diego, Cambridge, Oxford.

Jeon, M. 2017b. Emotions and Affect in Human Factors and Human-Computer Interaction: Taxonomy, Theories, Approaches, and Methods. In Emotions and Affect in Human Factors and Human-Computer Interaction, Pages 3-26, Mounghoon Jeon, eds., Academic Press, London, San Diego, Cambridge, Oxford.

Jifu Lan. 2004. 蓝吉福. eds. 禅宗全书. published by 北京图书馆出版社.

Jue Zhang. 2012. 张觉, 荷子译注, published by 上海古籍出版社.

Kejian Huang. 2012. 黄克剑. eds. 公孙龙子（外三种）, published by 中华书局.

McDuff, D., Kaliouby, R., Senechal, T., Amr, M., Cohn, J. \& Picard, R. 2013. Affectiva-MIT facial expression dataset (AM-FED): Naturalistic and spontaneous facial expressions collected In-the-Wild, Proc. IEEE Conf. Comput. Vis. Pattern Recognition. Workshops, pp. 881-888.

Microsoft. 2021. The Microsoft Emotion API. Website. Available from: https: //azure.microsoft.com/enus/services/cognitive-services/emotion.

Ong, D. C. et al. 2021. Modeling Emotion in Complex Stories: The Stanford Emotional Narratives Dataset, in IEEE Transactions on Affective Computing, vol. 12, no. 3, pp. 579-594, 1 July-Sept.

Picard, R. 2010. Affective computing: from laughter to IEEE. IEEE Transactions on Affective Computing, $1(1), 11-17$.

Poria, S., Cambria, E., Bajpai, R. \& Hussain, A. 2017. A review of affective computing: From unimodal analysis to multimodal fusion, Inf. Fusion, vol. 37, pp. 98-125.

Reisenzein, R. et al. 2013. Computational modeling of emotion: toward improving the inter and intradisciplinary exchange. IEEE Transactions on Affective Computing, 4 (3), 246-266.

Ringeval, F., Sonderegger, A., Sauer, J. \& Lalanne, D. 2013. Introducing the RECOLA multimodal corpus of remote collaborative and affective interactions, Proc. IEEE Int. Conf. Workshops Autom. Face Gesture Recognit., pp. 1-8, 2013.

Sariyanidi, E., Gunes, H. \& Cavallaro, A. 2015. Automatic analysis of facial affect: A survey of registration representation and recognition, IEEE Trans. Pattern Anal. Mach. Intell., vol. 37, no. 6, pp. 1113-1133.

Scheutz, M. 2012. The Affect Dilemma for Artificial Agents: Should We Develop Affective Artificial Agents? IEEE Transactions on Affective Computing, Vol. 3, No. 4.

Schuller, B. \& Batliner, A. 2013. Computational Paralinguistics: Emotion Affect and Personality in Speech and Language Processing, Hoboken, NJ, USA:Wiley, 2013.

Searle, J. R. 1983. Intentionality. Cambridge University Press, Cambridge, New York, Melbourne.

Searle, J. R. 2004 Mind: A Brief Introduction. Oxford University Press, New York.

Shangkuan Rao. 2016. 饶尚宽. eds. 老子, published by 中华书局.

Shapiro, L. 2014. The Routledge handbook of embodied cognition. Routledge. 